MODELING, IDENTIFICATION AND CONTROL, 1980, VOL. 1, NO. 2, 105-117

doi: $10.4173 /$ mic.1980.24

\title{
A finite element solution of the Navier-Stokes equations for two-dimensional and axis-symmetric flow
}

\author{
S. Ø. WILLE†
}

Keywords: Finite element method, blood flow, arteries.

The finite element formulation of the Navier-Stokes equations is derived for two-dimensional and axis-symmetric flow. The simple triangular, T6, isoparametric element is used. The velocities are interpolated by quadratic polynomials and the pressure is interpolated by linear polynomials. The non-linear simultaneous equations are solved iteratively by the Newton-Raphson method and the element matrix is given in the Newton-Raphson form. The finite element domain is organized in substructures and an equation solver which works on each substructure is specially designed. This equation solver needs less storage in the computer and is faster than the traditional banded equation solver. To reduce the amount of input data an automatic mesh generator is designed. The input consists of the coordinates of eight points defining each substructure with the corresponding boundary conditions. In order to interpret the results they are plotted on a calcomp plotter. Examples of plots of the velocities, the streamlines and the pressure inside a two-dimensional flow divider and an axis-symmetric expansion of a tube are shown for various Reynolds numbers.

\section{Introduction}

The solution of the Navier-Stokes equations forms an important field in fluid dynamics. The analytic solutions of these equations are limited to regular geometries where the fluid behaviour is relatively simple. The finite difference method has been extensively used to solve the Navier-Stokes equations numerically. However, the finite difference method can only be applied when the geometry of the fluid domain can be modelled by rectangles. The finite difference method has limitations when severe non-linearities are introduced into the equations.

The finite element method has been derived and applied to some problems concerning fluid flow (Hood and Taylor 1974) and has been shown to be superior to the finite difference method (Zienkiewics 1971, Taylor and Hood 1973). At present there exists several formulations of the Navier-Stokes equations. The formulation chosen in this work is the one given by Hood and Taylor (1974).

In most of the literature on the subject the finite element equations are only given explicitly. To obtain solutions for non-linear problems the Newton-Raphson method is most commonly applied and the finite element equations are therefore given in this form.

In the present work special attention has been paid to the equation solver. Finite element analysis requires rather large storage in the computer and long computer times. An efficient way of reducing the amount of fast memory is to use the substructuring technique. In this method only the data belonging to one substructure resides in the memory at one time while the data of the others are temporarily stored on disc files. As substructuring implies that the global element matrix becomes block diagonal a special equation solver is designed.

\section{† Institute of Informatics, University of Oslo, Norway.}


The large amount of input data, as well as output data, in finite element analysis has led to the construction of both a pre- and post-data processor to reduce the possibility of introducing errors in the finite element models. The pre-processor reduces the input data considerably and the post-processor converts the numerical output into graphical plots.

In this present paper the finite element method is demonstrated by solving the Navier-Stokes equations for a two-dimensional flow divider and an axis-symmetrical expansion tube. It is hoped that the method would have a particular application in the analysis of flow and pressure patterns in the cardiovascular system.

\section{Mesh generation}

The input data to the pre-processor consists of the coordinates of eight nodes which define a quadratic rectangular substructure and the boundary conditions on each side of the substructure. These nodes are situated at the corners and sides of the substructure, thus permitting curved boundaries. The coarseness of each substructure is defined by two parameters which specify the number of divisions wanted in both directions. Figure 1 shows an example of substructuring and the mapping used to obtain curved sides. Denoting the quadratic rectangular shape functions as $M_{i}$ the coordinates of the substructure are defined by

$$
\begin{aligned}
& x=\sum M_{i} x_{i} \\
& y=\sum M_{i} y_{i}
\end{aligned}
$$

where $x_{i}$ and $y_{i}$ are the coordinates of the eight nodes. The substructuring technique has been used for some time in mechanics. The way of substructuring described here is designed to suit hydrodynamic problems.
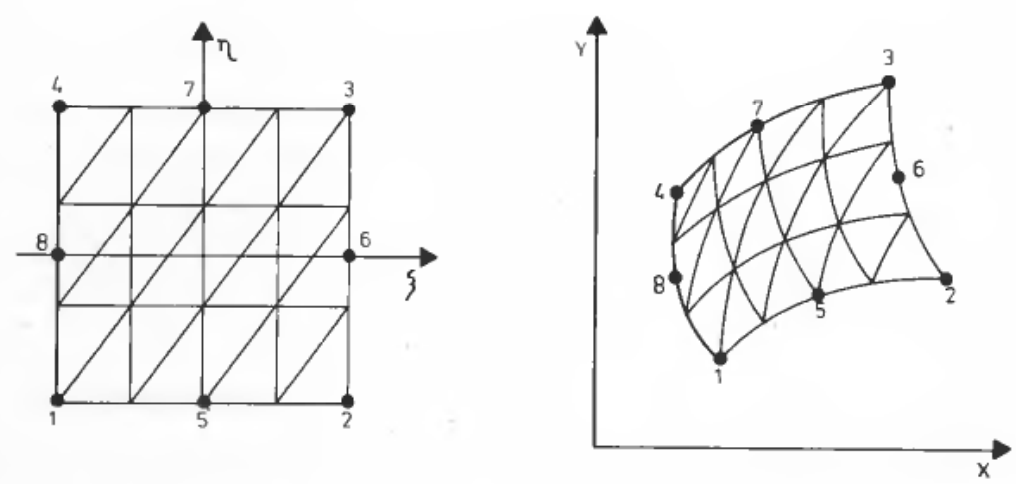

Figure 1. The mapping and division of a substructure by isoparametric elements.

\section{The Navier-Stokes equations}

The equations governing two-dimensional and axis-symmetric steady flow are the well-known Navier-Stokes equations (Sabersky et al. 1971). In the two-dimensional case these are

$$
\frac{1}{\rho} \frac{\partial p}{\partial x}-\mu\left(\frac{\partial^{2} u}{\partial x^{2}}+\frac{\partial^{2} u}{\partial y^{2}}\right)+u \frac{\partial u}{\partial x}+v \frac{\partial u}{\partial y}=0
$$




$$
\begin{gathered}
\frac{1}{\rho} \frac{\partial p}{\partial y}-\mu\left(\frac{\partial^{2} v}{\partial x^{2}}+\frac{\partial^{2} v}{\partial y^{2}}\right)+u \frac{\partial v}{\partial x}+v \frac{\partial v}{\partial y}=0 \\
\frac{\partial u}{\partial x}+\frac{\partial v}{\partial y}=0
\end{gathered}
$$

In the axis-symmetric case these equations take the form

$$
\begin{gathered}
\frac{1}{\rho} \frac{\partial p}{\partial x}-\mu\left(\frac{1}{r} \frac{\partial u}{\partial r}+\frac{\partial^{2} u}{\partial x^{2}}+\frac{\partial^{2} u}{\partial r^{2}}\right)+u \frac{\partial u}{\partial x}+v \frac{\partial u}{\partial r}=0 \\
\frac{1}{\rho} \frac{\partial p}{\partial y}-\mu\left(\frac{1}{r} \frac{\partial v}{\partial r}-\frac{v}{r^{2}}+\frac{\partial^{2} v}{\partial x^{2}}+\frac{\partial^{2} v}{\partial r^{2}}\right)+u \frac{\partial v}{\partial x}+v \frac{\partial v}{\partial r}=0 \\
\frac{v}{r}+\frac{\partial u}{\partial x}+\frac{\partial v}{\partial r}=0
\end{gathered}
$$

where $u=$ velocity in the $x$ direction, $v=$ velocity in $y$ or $r$ direction, $p=$ pressure, $\rho=$ density and $\mu=$ kinematic viscosity. The flow described by these equations is assumed to be laminar, steady and isothermal and the fluid homogeneous, incompressible and Newtonian.

\section{The finite element formulation}

There exist several ways of deriving the finite elements form of the Navier-Stokes equations. The one used in this work is the pressure-velocity formulation with the Galerkin method to form the equations. The main advantage of this formulation is that only velocity needs to be specified on the external boundaries. Let $N_{i}$ denote the quadratic shape functions for the velocities and $L_{i}$ the linear shape functions for the pressure. The velocities and pressure over a triangular element can then be expressed as

$$
\begin{aligned}
& u=\sum N_{i} u_{i} \\
& v=\sum N_{i} v_{i} \\
& p=\sum L_{i} p_{i}
\end{aligned}
$$

where $u_{i}, v_{i}$ and $p_{i}$ are the nodal values of velocities and pressure. Inserting these expressions into the Navier-Stokes equations and applying the Galerkin method, the finite element equations take the following forms:

Two-dimensional flow:

$$
\begin{aligned}
& F_{1 i}=\iint_{e}[\mu\left(\frac{\partial N_{i}}{\partial x} \sum \frac{\partial N_{k}}{\partial x} u_{k}+\frac{\partial N_{i}}{\partial y} \sum \frac{\partial N_{k}}{\partial y} u_{k}\right)+N_{i}\left[\left(\sum N_{k} u_{k}\right) \sum \frac{\partial N_{k}}{\partial x} u_{k}\right. \\
&\left.\left.+\left(\sum N_{k} v_{k}\right) \sum \frac{\partial N_{k}}{\partial y} u_{k}+\frac{1}{\rho} \sum \frac{\partial L_{k}}{\partial x} p_{k}\right]\right] d x d y-\int_{s} \mu N_{i} \sum \frac{\partial N_{k}}{\partial n} u_{k} d s=0 \\
& F_{2 i}=\iint_{e}\left[\mu\left(\frac{\partial N_{i}}{\partial x} \sum \frac{\partial N_{k}}{\partial x} v_{k}+\frac{\partial N_{i}}{\partial y} \sum \frac{\partial N_{k}}{\partial y} v_{k}\right)+N_{i}\left[\left(\sum N_{k} u_{k}\right) \sum \frac{\partial N_{k}}{\partial x} v_{k}\right.\right. \\
&\left.\left.+\left(\sum N_{k} v_{k}\right) \sum \frac{\partial N_{k}}{\partial y} v_{k}+\frac{1}{\rho} \sum \frac{\partial L_{k}}{\partial y} p_{k}\right]\right] d x d y-\int_{s} \mu N_{i} \sum \frac{\partial N_{k}}{\partial n} v_{k} d s=0
\end{aligned}
$$




$$
F_{3 i}=\iint_{e} L_{i}\left(\sum \frac{\partial N_{k}}{\partial x} u_{k}+\sum \frac{\partial N_{k}}{\partial y} v_{k}\right) d x d y=0
$$

Axis-symmetric flow:

$$
\begin{aligned}
& F_{1 i}=\iint_{e} {\left[\mu\left(-\frac{N_{i}}{r} \sum \frac{\partial N_{k}}{\partial r} u_{k}+\frac{\partial N_{i}}{\partial x} \sum \frac{\partial N_{k}}{\partial x} u_{k}+\frac{\partial N_{i}}{\partial r} \sum \frac{\partial N_{k}}{\partial r} u_{k}\right)\right.} \\
&+\left.N_{i}\left[\left(\sum N_{k} u_{k}\right) \sum \frac{\partial N_{k}}{\partial x} u_{k}+\left(\sum N_{k} v_{k}\right) \sum \frac{\partial N_{k}}{\partial r} u_{k}+{ }_{\rho}^{1} \sum \frac{\partial L_{k}}{\partial x} p_{k}\right]\right] d x d r \\
& F_{2 i}=\iint_{e}\left[\mu \left(-\frac{N_{i}}{r} \sum \frac{\partial N_{k}}{\partial r} v_{k}+\frac{N_{i}}{r^{2}} \sum N_{i} \sum \frac{\partial N_{k}}{\partial n} u_{k} d s\right.\right. \\
&+N_{i}\left[\left(\sum N_{k} u_{k}\right) \sum \frac{\partial N_{i}}{\partial x} \sum \frac{\partial N_{k}}{\partial x} v_{k}+\frac{\partial N_{i}}{\partial r} \sum \frac{\partial N_{k}}{\partial r} v_{k}\right) \\
&\left.\left.F_{3 i}=\iint_{e} L_{i}\left(\frac{1}{r} \sum N_{k} v_{k}\right) \sum \frac{\partial N_{k}}{\partial r} v_{k}+\frac{1}{\rho} \sum \frac{\partial L_{k}}{\partial r} p_{k}\right]\right] d x d r
\end{aligned}
$$

Differentiating and ordering the equations according to the Newton-Raphson method, the equation system takes the form

$$
\left[\begin{array}{lll}
\frac{\partial F_{1 i}}{\partial u_{j}}, & \frac{\partial F_{1 i}}{\partial v_{j}}, & \frac{\partial F_{1 i}}{\partial p_{j}} \\
\frac{\partial F_{2 i}}{\partial u_{j}}, & \frac{\partial F_{2 i}}{\partial v_{j}}, & \frac{\partial F_{2 i}}{\partial p_{j}} \\
\frac{\partial F_{3 i}}{\partial u_{j}}, & \frac{\partial F_{3 i}}{\partial v_{j}}, & \frac{\partial F_{3 i}}{\partial p_{j}}
\end{array}\right]\left[\begin{array}{c}
\Delta u_{j} \\
\Delta v_{j} \\
\Delta p_{j}
\end{array}\right]=-\left[\begin{array}{l}
F_{1 i} \\
F_{2 i} \\
F_{3 i}
\end{array}\right]
$$

Two-dimensional flow:

$$
\begin{aligned}
\frac{\partial F_{1 i}}{\partial u_{j}}=\iint_{e}\left[\mu\left(\frac{\partial N_{i}}{\partial x} \frac{\partial N_{j}}{\partial x}+\frac{\partial N_{i}}{\partial y} \frac{\partial N_{j}}{\partial y}\right)+\right. & N_{i}\left(N_{j} \sum \frac{\partial N_{k}}{\partial x} u_{k}+\frac{\partial N_{j}}{\partial x} \sum N_{k} u_{k}\right. \\
& \left.\left.+\frac{\partial N_{j}}{\partial y} \sum N_{k} v_{k}\right)\right] d x d y-\int_{s} \mu N_{i} \frac{\partial N_{j}}{\partial n} d s \\
\frac{\partial F_{1 i}}{\partial v_{j}} & =\iint_{e} N_{i} N_{j} \sum \frac{\partial N_{k}}{\partial y} u_{k} d x d y \\
\frac{\partial F_{1 i}}{\partial p_{j}} & =\iint_{e} \frac{N_{i}}{\rho} \frac{\partial L_{j}}{\partial x} d x d y \\
\frac{\partial F_{2 i}}{\partial u_{j}} & =\iint_{e} N_{i} N_{j} \sum \frac{\partial N_{k}}{\partial x} v_{k} d x d y
\end{aligned}
$$




$$
\begin{gathered}
\frac{\partial F_{2 i}}{\partial v_{j}}=\iint_{e}\left[\mu\left(\frac{\partial N_{i}}{\partial x} \frac{\partial N_{j}}{\partial x}+\frac{\partial N_{i}}{\partial y} \frac{\partial N_{j}}{\partial y}\right)+N_{i}\left(N_{j} \sum \frac{\partial N_{k}}{\partial y} v_{k}+\frac{\partial N_{j}}{\partial x} \sum N_{k} u_{k}\right.\right. \\
\left.\left.\quad+\frac{\partial N_{j}}{\partial y} \sum N_{k} v_{k}\right)\right] d x d y-\int \mu N_{i} \frac{\partial N_{j}}{\partial n} d s \\
\frac{\partial F_{2 i}}{\partial p_{j}}=\iint_{e} \frac{N_{i}}{\rho} \frac{\partial L_{j}}{\partial y} d x d y \\
\frac{\partial F_{3 i}}{\partial u_{j}}=\iint_{e} L_{i} \frac{\partial N_{j}}{\partial x} d x d y \\
\frac{\partial F_{3 i}}{\partial v_{j}}=\iint_{e} L_{i} \frac{\partial N_{j}}{\partial y} d x d y \\
\frac{\partial F_{3 i}}{\partial p_{j}}=0
\end{gathered}
$$

Axis-symmetric flow:

$$
\begin{aligned}
& \frac{\partial F_{1 i}}{\partial u_{j}}=\iint_{\boldsymbol{e}}\left[\mu\left(-\frac{N_{i}}{r} \frac{\partial N_{j}}{\partial r}+\frac{\partial N_{i}}{\partial x} \frac{\partial N_{j}}{\partial x}+\frac{\partial N_{i}}{\partial r} \frac{\partial N_{j}}{\partial r}\right)+N_{i}\left(N_{j} \sum \frac{\partial N_{k}}{\partial x} u_{k}\right.\right. \\
& \left.\left.+\frac{\partial N_{j}}{\partial x} \sum N_{k} u_{k}+\frac{\partial N_{j}}{\partial r} \sum N_{k} v_{k}\right)\right] d x d r-\int_{s} \mu N_{i} \frac{\partial N_{j}}{\partial n} d s \\
& \frac{\partial F_{1 i}}{\partial v_{j}}=\iint_{e} N_{i} N_{j} \sum \frac{\partial N_{k}}{\partial r} u_{k} d x d r \\
& \frac{\partial F_{1 i}}{\partial p_{j}}=\iint_{e} \frac{N_{i}}{\rho} \frac{\partial L_{j}}{\partial x} d x d r \\
& \frac{\partial F_{2 i}}{\partial u_{j}}=\iint_{e} N_{i} N_{j} \sum \frac{\partial N_{k}}{\partial x} v_{k} d x d r \\
& \frac{\partial F_{2 i}}{\partial v_{j}}=\iint_{e}\left[\mu\left(-\frac{N_{i}}{r} \frac{\partial N_{j}}{\partial r}+\frac{N_{i}}{r^{2}} N_{j}+\frac{\partial N_{l}}{\partial x} \frac{\partial N_{j}}{\partial x}+\frac{\partial N_{i}}{\partial r} \frac{\partial N_{j}}{\partial r}\right)+N_{i}\left(N_{j} \sum \frac{\partial N_{k}}{\partial r} v_{k}\right.\right. \\
& \left.\left.+\frac{\partial N_{j}}{\partial x} \sum N_{k} u_{k}+\frac{\partial N_{j}}{\partial r} \sum N_{k} v_{k}\right)\right] d x d r-\int_{s} \mu N_{i} \frac{\partial N_{j}}{\partial n} d s \\
& \frac{\partial F_{2 i}}{\partial p_{j}}=\iint_{e} \frac{N_{i}}{\rho} \frac{\partial L_{j}}{\partial r} d x d r \\
& \frac{\partial F_{3 i}}{\partial u_{j}}=\iint_{e} L_{i} \frac{\partial N_{j}}{\partial x} d x d r \\
& \frac{\partial F_{3 i}}{\partial v_{j}}=\iint_{e} L_{i}\left(\frac{N_{j}}{r}+\frac{\partial N_{j}}{\partial r}\right) d x d r \\
& \frac{\partial F_{3 i}}{\partial p_{j}}=0
\end{aligned}
$$


Applying the Newton-Raphson solution technique, the correction of the solution vector is obtained at each step. Thus,

$$
\begin{aligned}
& u_{j}=u_{j}{ }^{1}+\Delta u_{j} \\
& v_{j}=v_{j}{ }^{1}+\Delta v_{j} \\
& p_{j}=p_{j}{ }^{1}+\Delta p_{j}
\end{aligned}
$$

where $u_{j}{ }^{1}, v_{j}{ }^{1}$ and $p_{j}{ }^{1}$ are the solutions obtained at the previous iteration. The convergence criteria chosen is

$$
\begin{gathered}
\epsilon_{v}=\left(\frac{\max \left(\Delta u^{2}+\Delta v^{2}\right)}{\max \left(u^{2}+v^{2}\right)}\right)^{1 / 2} \\
\epsilon_{p}=\frac{\max |\Delta p|}{\max |p|}
\end{gathered}
$$

When the relative errors $\epsilon_{v}$ and $\epsilon_{p}$ are sufficiently small, the iteration is stopped. Typical values of $\epsilon_{v}$ and $\epsilon_{p}$ are $10^{-4}$. The speed of the convergence depends on the closeness of the start vector to the final solution value, but more than five iterations are seldom necessary. To obtain solutions at higher Reynolds numbers the solution at a lower Reynolds number is used as start vector and the Reynolds number increased by lowering the kinematic viscosity.

When the velocity field is found the streamlines are calculated by the following equations

Two dimensional flow:

The streamfunction is defined by

$$
u=-\frac{\partial \psi}{\partial y}, \quad v=\frac{\partial \psi}{\partial x}
$$

Differentiating these expressions and applying the Galerkin process the following equation system is obtained

$$
\left[\iint_{e}\left(\frac{\partial N_{i}}{\partial x} \frac{\partial N_{j}}{\partial x}+\frac{\partial N_{i}}{\partial y} \frac{\partial N_{j}}{\partial y}\right) d x d y\right] \psi_{j}=\int_{s} N_{i} \frac{\partial \psi}{\partial n} d s-\iint_{e} N_{i}\left(\frac{\partial v}{\partial x}-\frac{\partial u}{\partial y}\right) d x d y
$$

Axis-symmetric flow:

The streamfunction is defined by

$$
u=-\frac{1}{r} \frac{\partial \psi}{\partial r}, \quad v=\frac{1}{r} \frac{\partial \psi}{\partial x}
$$

The streamfunction equation becomes

$$
\begin{aligned}
{\left[\iint_{e}\left(\frac{\partial N_{i}}{\partial x} \frac{\partial N_{j}}{\partial x}+\frac{\partial N_{i}}{\partial r} \frac{\partial N_{j}}{\partial r}\right) d x d r\right] \psi_{j}=\int_{s} N_{i} \frac{\partial \psi}{\partial n} d s } & \\
& \quad-\iint_{e} N_{i}\left(r \frac{\partial v}{\partial x}-r \frac{\partial u}{\partial r}-u\right) d x d r
\end{aligned}
$$

These equations are linear and easily solved. 


\section{Block diagonal equation solver}

As the entire finite element domain is divided into substructures, a local element matrix corresponding to each substructure can be calculated. Figure 2 shows two examples of substructuring and the local numbering of the nodes in each substructure. Local numbering of the nodes has to be made so that the highest numbers are found on the border to the proceeding substructure. The size of the global element matrix of the entire flow domain will be of a size equal to the total number of degrees of freedom. Substructuring implies that the global element matrix can be replaced by three smaller local element matrices corresponding to each substructure.

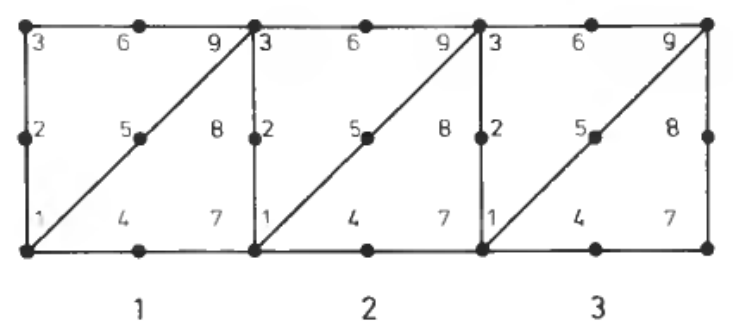

3

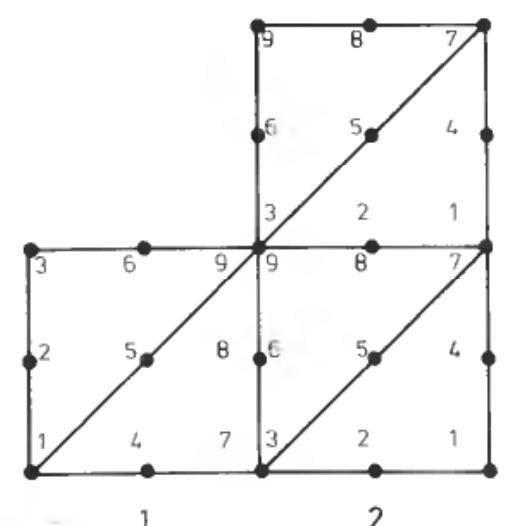

Figure 2. Examples of substructure linking.

Figure 3 shows the global element matrix and the local element matrices of the substructures. The dotted lines show the corresponding banded matrix. The area outside the submatrices contains only zeros. From the figure it is seen that the banded matrix needs less storage than the global matrix and that the submatrices need less storage than the banded matrix. An additional disadvantage of a banded equation solver is a temporary extra storage of half the bandwidth, which is needed during the elimination. When designing each substructure it is important that this is done in a way that leads to the fewest possible zeros in the local matrices. In this way both storage and computer time are reduced.

The local element matrices contain internal and external degrees of freedom. The external degrees of freedom are those of the nodes connected to the proceeding 


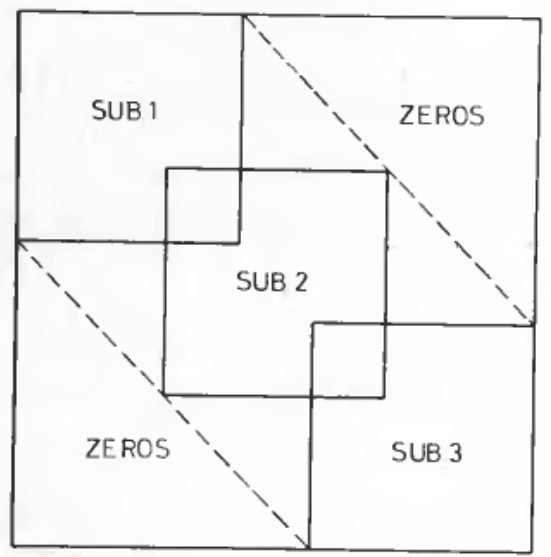

Figure 3. The global element matrix with the local element matrices situated on the diagonal. The dotted lines indicate the bandwidth of the global element matrix.

substructure. The internal degrees of freedom are those of the nodes which are not contained in the proceeding substructure.

The element matrix of each substructure may then be divided into four parts as in Fig. 4. Here the submatrix $A_{\mathrm{ii}}$ represents the internal degrees of freedom. $A_{\mathrm{ic}}$ and $A_{\mathrm{ei}}$ are the coefficients associated with the connection of internal and external degrees of freedom. $A_{\mathrm{ee}}$ represents the external degrees of freedom. Similarly $X_{\mathrm{i}}$ and $B_{\mathrm{i}}, X_{\mathrm{e}}$ and $B_{\mathrm{e}}$ represent the internal and external degrees of freedom in the solution vector and the right-hand side. The elimination procedure is started by eliminating the internal degrees of freedom. The algorithm replaces $A_{\mathrm{ee}}$ and $B_{\mathrm{e}}$ by

$$
\begin{aligned}
\bar{A}_{\mathrm{ec}} & =A_{\mathrm{ee}}-A_{\mathrm{ei}} A_{\mathrm{ii}}{ }^{-1} A_{\mathrm{ie}} \\
\bar{B}_{\mathrm{c}} & =B_{\mathrm{e}}-A_{\mathrm{ei}} A_{\mathrm{ii}}{ }^{-1} B_{\mathrm{i}}
\end{aligned}
$$

$A_{\mathrm{ii}}{ }^{-1} A_{\mathrm{ie}}$ is calculated by solving the equation system with $A_{\mathrm{ii}}$ as coefficients and the columns in $A_{\text {ie }}$ as right-hand sides. The matrix inversion of $A_{\mathbf{i i}}$, which is a time consuming process, is thereby avoided. The next step is to add $A_{\mathrm{ee}}$ and $B_{\mathrm{e}}$ to the corresponding coefficients in the element matrix and the right-hand side vector of the proceeding substructure. The following substructure is eliminated in the same way.

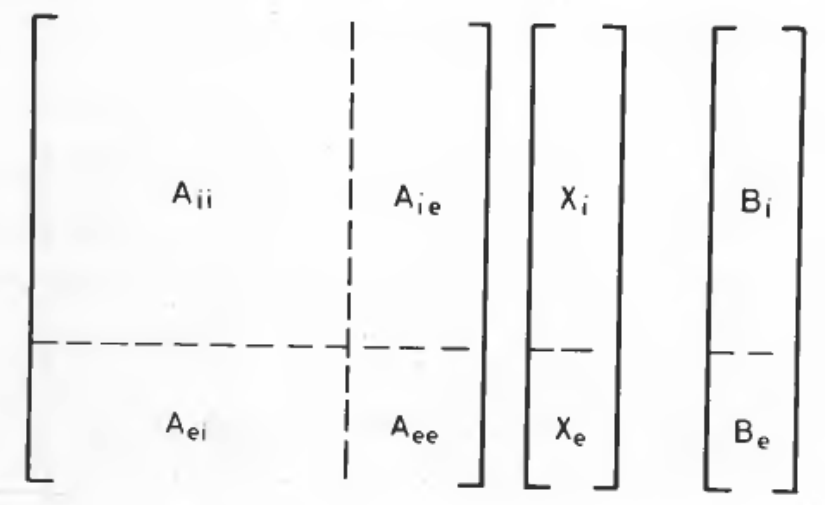

Figure 4. A local element matrix with submatrices of external and internal degrees of freedom. 
In the backward substitution, the solutions of the common degrees of freedom from the proceeding substructure are substituted into $X_{\mathrm{e}}$. The right-hand side is then replaced by

$$
\bar{B}_{\mathrm{i}}=B_{\mathrm{i}}-A_{\mathrm{ii}} X_{\mathrm{e}}
$$

The equations to be solved for this substructure are now

$$
A_{\mathrm{ii}} X_{\mathrm{i}}=\bar{B}_{\mathrm{i}}
$$

and the complete solution is found. The algorithm for linking several substructures follow the same procedure.

\section{Numerical examples}

\section{Two-dimensional case}

As an example of the two-dimensional analysis a flow divider may be considered. It consists of a tube dividing into two identical branches of total diameter equal to that of the input tube. As the flow divider is symmetrical it is only necessary to simulate the upper half. The mesh chosen for the divider is shown in Fig. 5. The flow domain has been divided into twenty substructures. The inlet boundary conditions are a parabolic velocity profile in the longitudinal direction $\left(u=0 \cdot 2\left(1-y^{2} / R^{2}\right)\right)$ and zero velocity in the transverse direction $(v=0)$. On the wall both the velocities are zero $(u=0, v=0)$ to fulfil the no-slip condition. At the outlet the normal derivatives of the velocities are zero $(\partial u / \partial n=0, \partial v / \partial n=0)$ and along the line of symmetry in the main tube the normal velocity $(v=0)$ and the normal derivative of the tangential velocity $(\partial u / \partial n=0)$ are zero. The pressure is specified to be zero at one node at the outlet wall.

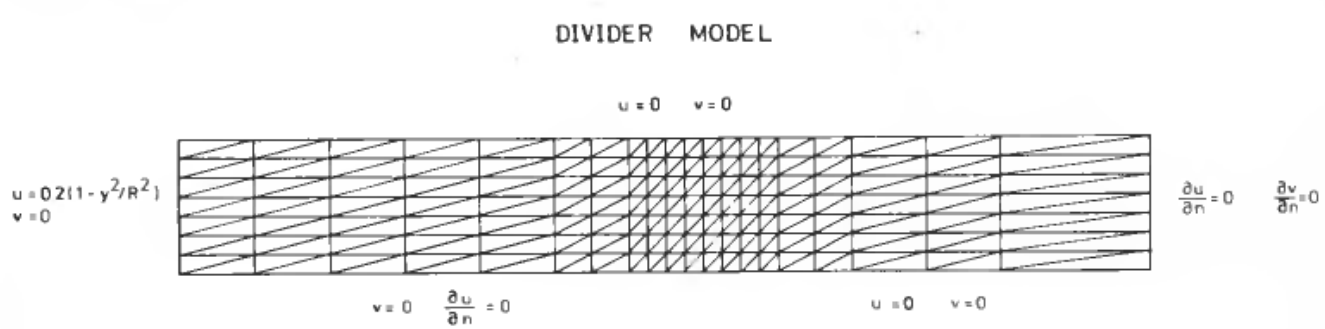

Figure 5. The mesh and boundary conditions of the flow divider. Each substructure consists of the triangular elements between the vertical lines. The inlet radius is $0.01 \mathrm{~m}$.

The results of the simulation for Reynolds number 100 are shown in Fig. 6 . In Fig. 7 the pressures along the outer wall and the line of symmetry are plotted separately.

\section{Axis-symmetrical case}

As an example of the axis-symmetrical analysis an axial expansion of a tube may be considered. The equivalent finite element representation is shown in Fig. 8. The mesh consists of eighteen substructures and isoparametric elements are used to describe the boundary. The boundary conditions are indicated on the figure and they are of the same kind as for the flow divider. The results of the simulations for Reynolds number 10 and 200 are shown in Figs. 9-12. 
PRESSURE

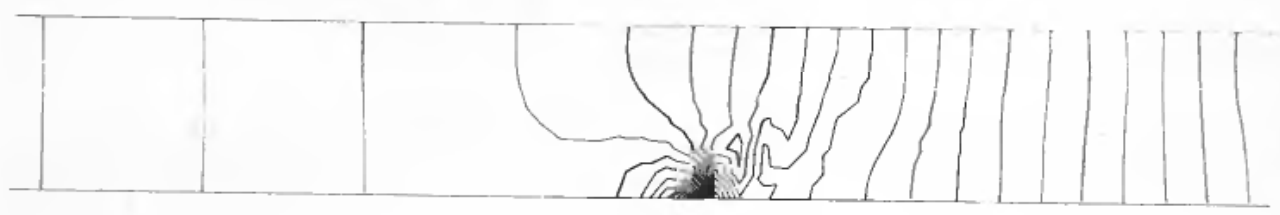

$\Delta p=10$

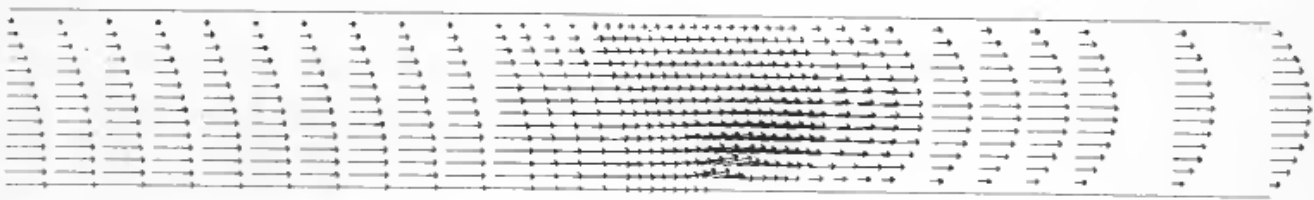

Reynolds number 100

Figure 6. The results of the simulation of the flow divider for Reynolds number 100 . The pressure plot shows isobars and the streamline plot shows equally spaced streamlines. The velocity plot shows the direction and magnitude of the velocities. The mean inlet velocity is $0.133 \mathrm{~m} \mathrm{~s}^{-1}$ and the viscosity is $2.67 \times 10^{-5} \mathrm{~m}^{2} \mathrm{~s}^{-1}$.

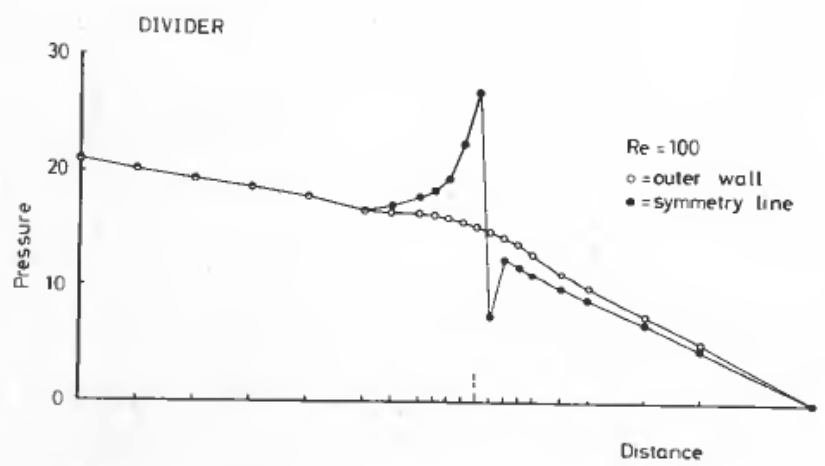

Figure 7. The pressure along the outer wall and the line of symmetry for Reynolds number 100. The apex of the flow divider is indicated by a dashed line in the abscissa. 


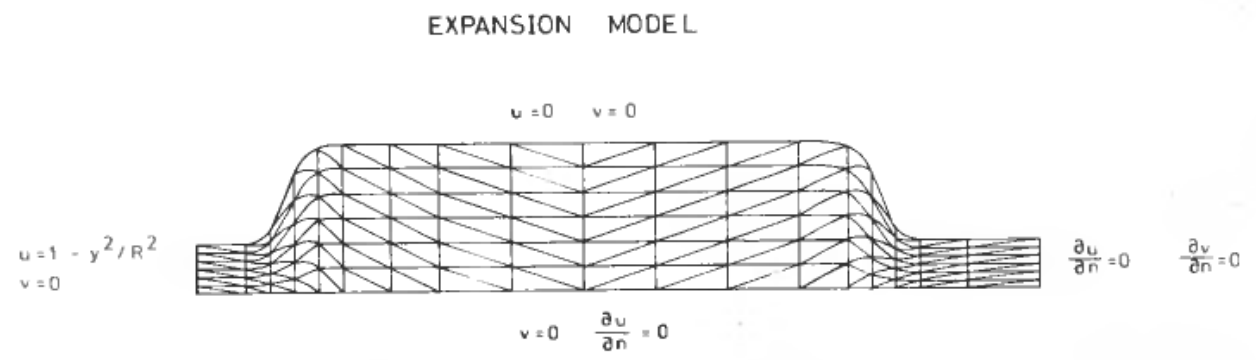

Fig. 8. The mesh and boundary conditions of the tube expansion. Each substructure consists of the triangular element between the vertical lines. The inlet radius is $0.01 \mathrm{~m}$.

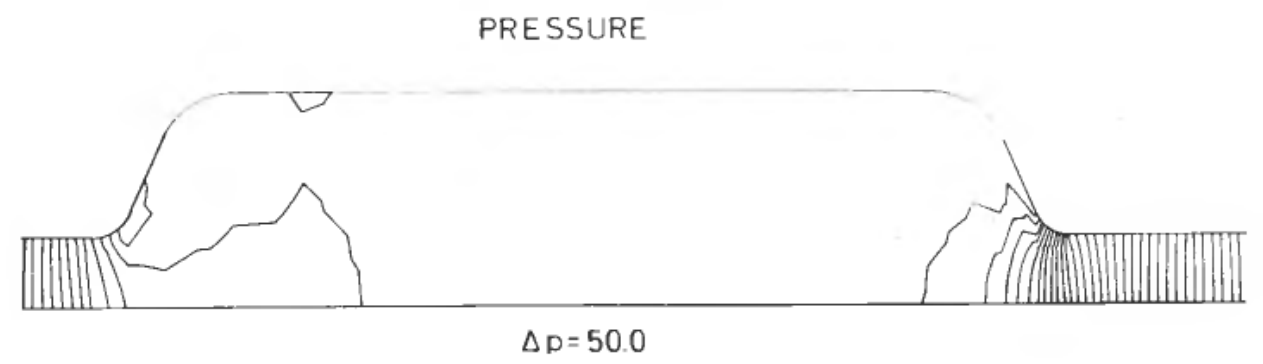

STREAMLINES

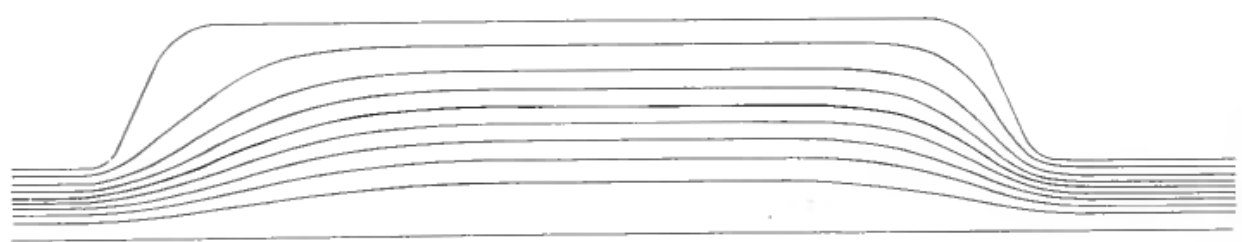

VELOCITY

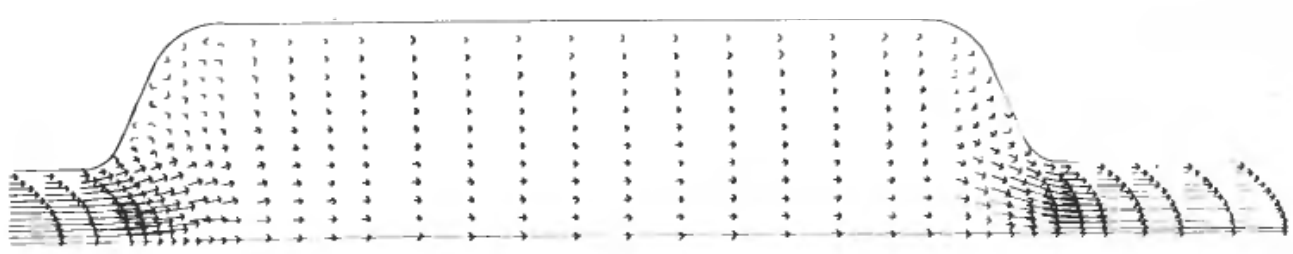

Reynolds number 10

Figure 9. The results of the simulation of the expansion for Reynolds number 10. The mean inlet velocity is $0.5 \mathrm{~m} \mathrm{~s}^{-1}$ and the viscosity is $1 \times 10^{-3} \mathrm{~m}^{2} \mathrm{~s}^{-1}$. 


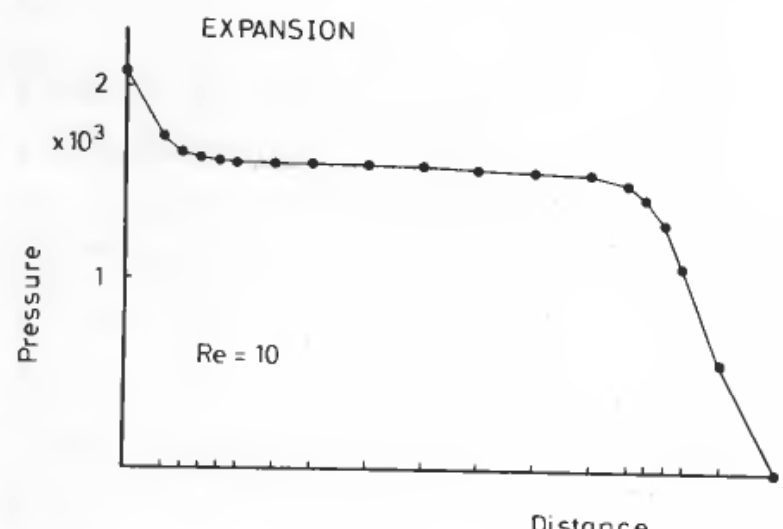

Figure 10. The pressure along the symmetry axis in the expansion model.

PRESSURE

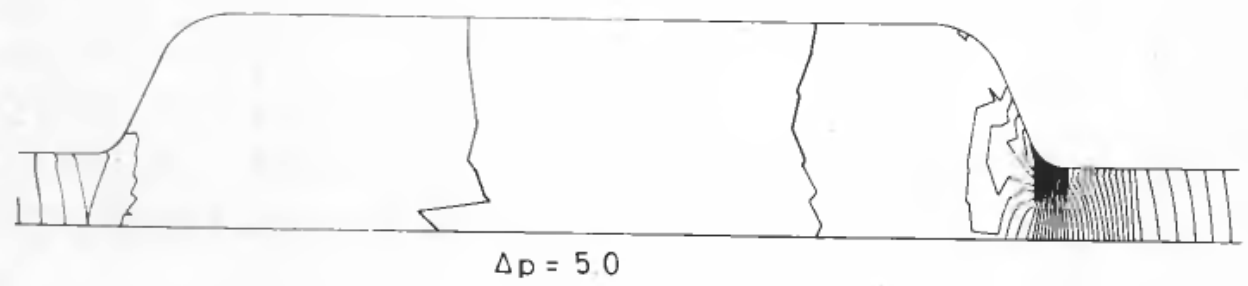

STREAMLINES

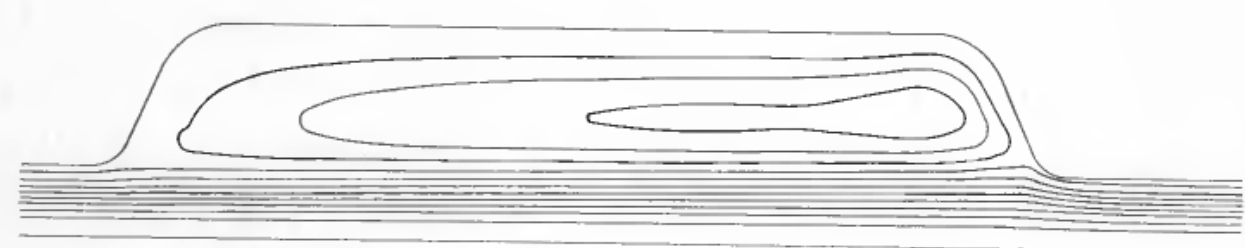

VELOCITY

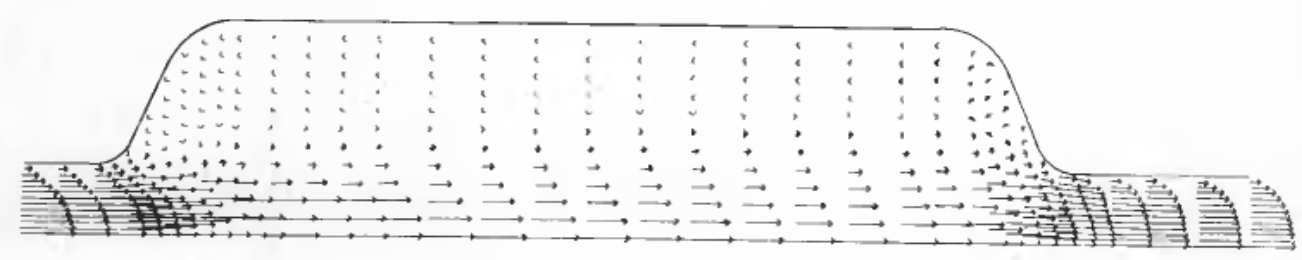

Reynolds number 200

Figure 11. The results of the simulation for the expansion for Reynolds number 200. The mean inlet velocity is $0.5 \mathrm{~m} \mathrm{~s}^{-1}$ and the viscosity is $5 \times 10^{-5} \mathrm{~m}^{2} \mathrm{~s}^{-1}$. 


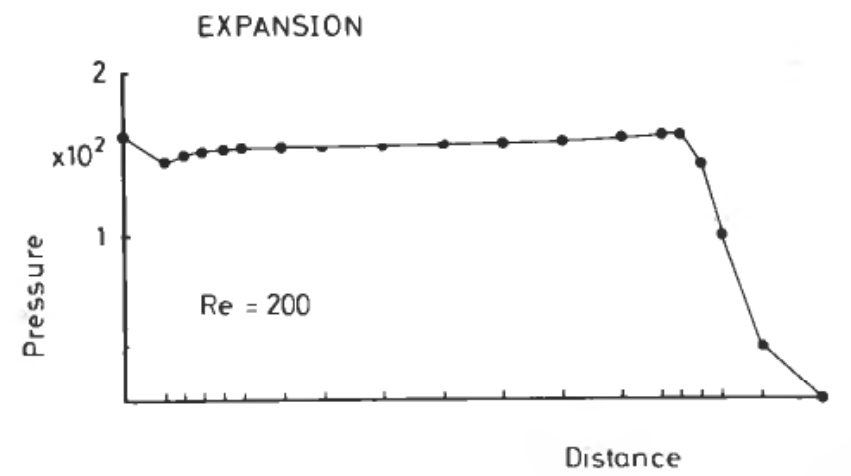

Figure 12. The pressure along the symmetry axis in the expansion model.

\section{Conclusion}

This paper has dealt with a finite element method to analyse two-dimensional and axis-symmetric flow. The discretization procedure has been based on the Gallerkin approach. The shape functions used are the complete quadratic polynomials for the velocities and linear polynomials for the pressure. The solution method chosen is the Newton-Raphson method and the equation system is expressed in this form.

A substructuring technique has been described and compared to the traditional finite element formulation. A block diagonal equation solver to suit the substructuring has been designed. The analysis has been carried out for two-dimensional and axissymmetrical configurations and the results presented in terms of flow and pressure patterns.

In future papers results obtained by this computer program on models of arterial systems will be presented and discussed from a physiological point of view.

\section{ACKNOWLEDGMENTS}

The author is grateful to Pål Bergan, Olav Dahl and Lars Walløe for discussions and encouragement throughout the work.

\section{REFERENCES}

Hood, P., and TAYLOR, C. (1974). Navier-Stokes equations using mixed interpolation. Proceedings of the International Symposium on Finite Element Methods in Flow Problems, pp. 121-132.

Sabersky, R. H., Acosta, A. J., and Hauptmann, E. G. (1971). Fluid Flow. A First Course in Fluid Mechanics (Macmillan).

TAYLOR, C., and HoOD, P, (1973). A numerical solution of the Navier-Stokes equations using the finite element technique. Comput. Fluids, 1, 73-100.

ZIENKIEWICS, O. C. (1971). The Finite Element Method in Engineering Science, 2nd edition (McGraw-Hill). 